handovers at one London NHS site. The site composed 19 forensic secure psychiatric wards, an older adult psychiatric ward and the Home Treatment team.

Before the intervention, handovers were carried out via verbal communication between consecutive Core Trainees. A separate telephone handover took place between the Core Trainee, Speciality Registrar and Consultant on call. The primary driver of this project was the lack of quality communication at handovers. Our aim was to increase safety, efficacy, and accountability. Successful processes at other sites and trainee feedback was analysed. It was decided to implement an email handover system and to utilise MS teams for enhanced communication of verbal handovers.

One problem encountered was ensuring dissemination of the new handover protocols across all affected staff members. This problem was surmounted with regular communication of the changes.

Ongoing feedback showed approval ratings of over $83 \%$ amongst some trainees. Trainee feedback was used to continue to shape systems in place, to continue our PDSA cycle of change and improvement.

This solution illustrates how utilising simple technology can transform reliability and continuity of care. The project demonstrates the power of trainees to enact change using simple, practical solutions by using open dialogue and negotiation with the trust, which has increased trainee morale. In this way, a tailored solution specific to the needs of the medical staff was produced. The project also leaves a lasting legacy of introducing technology into the handover, which can continue to evolve as new technological advances arise.

\section{EXTENSION OF A UNIVERSITY BASED PERIOPERATIVE MEDICINE PROGRAM TO A DISTANT RURAL HOSPITAL}

${ }^{1}$ Robert Gould, ${ }^{2}$ Brandon Taylor, 'Michael Wall. 'Department of Anesthesiology University of Minnesota USA; ${ }^{2}$ Fairview Range Medical Center USA

10.1136/leader-2021-FMLM.23

Leadership at Fairview Range Medical Center initiated the project by approaching leadership of the University of Minnesota.

Department of Anesthesiology at the University negotiated a contract to provide medical directorship of the operating room and medical directorship of perioperative medicine services. The current medical directors of both services at the University of Minnesota were appointed dual roles in each site.

Medical Directors have monthly visits via scheduled air service to meet administrative, surgical and medical leadership.

Development of a robust tele medicine program to provide preoperative, intraoperative and postoperative anesthesia care with video technology.

Collaboration between rural hospitals and academic medical centers should facilitate provision of the best possible evidence based perioperative care for the largest surgical population. We have demonstrated the ability to use multiple platforms synergistically to collaborate on all facets of perioperative care. The cooperative venture has been well received by all providers and has energized care in both hospitals. Similar collaborations could greatly improve care and value for the $20 \%$ of the population that receives care at rural hospitals. While telemedicine has been described to assist preoperative assessment, this to the best of our knowledge is one of the only programs that offer real time intraoperative and postoperative anesthesia consultative management via tele medicine.

\section{EYESTRIVE: A VIRTUAL OPHTHALMOLOGY TASTER PROGRAMME FOR FOUNDATION DOCTORS LOOKING FOR AN INSIGHT INTO A CAREER IN OPHTHALMOLOGY DURING THE SARS-COV-2 PANDEMIC}

${ }^{1}$ Maha Noor*, ${ }^{2}$ Akash Dharni, ${ }^{3}$ Rehna Khan, ${ }^{4}$ Shabeena Aziz. ${ }^{1}$ Bradford Teaching Hospitals NHS Foundation Trust, Bradford, UK; ${ }^{2}$ Bradford Teaching Hospitals NHS Foundation Trust, Bradford, UK; ${ }^{3}$ Consultant Ophthalmologist, Evolutio Care Innovations Ltd, UK; ${ }^{4}$ General Practitioner, UK

\subsection{6/leader-2021-FMLM.24}

Background The implementation of social distancing measures during the SARS-CoV-2 pandemic limited Ophthalmology departments in offering face-to-face taster days to foundation doctors to promote career development. Therefore, we developed an e-learning platform for junior doctors to gain an insight into Ophthalmology, while maintaining the same level of detail as face-to-face programmes.

Methods We explored the literature surrounding medical education adaptations to social distancing measures. A pre-programme survey was conducted investigating interest in a virtual Ophthalmology taster programme within the Yorkshire and Humber deanery. A positive response resulted in the development of 'Eyestrive' - a comprehensive e-learning platform (www.eyestrive.org.uk). It consisted of five pre-course modules, each led by a consultant ophthalmologist, followed by a full day virtual interactive workshop comprising of lectures and wet lab. The modules included:

1. Clinical Skills: resources on ophthalmic examinations with follow-on feedback.

2. Clinical Signs: photographs of ocular pathologies complemented by multiple choice questions.

3. Special Module: a) Teaching Others b) Audit/Quality Improvement c) Critical Appraisal

4. FRCOphth Part 1 Club: key topics of the exam taught by trainees.

5. Virtual Wet Lab: attendees practiced suturing and knot tying techniques under supervision.

Results Twenty-four junior doctors attending from UK, Ireland, and Malaysia completed the programme. Pre- and post-programme questionnaires showed increased confidence in critical appraisal and surgical skills, understanding of quality improvement principles, and the ability to assess the acute red eye.

Conclusion Eyestrive sets a blueprint for designing a virtual training programme. The pre-course modules followed by 'virtual workshop' can be applied to undergraduate and postgraduate medical teaching across various specialties. In the COVID era, this has been a timely contribution.

\section{FIND A WAY OR MAKE ONE: A PROJECT TO IMPROVE THE HEALTH PLANNING PROCESS IN SRI LANKA}

${ }^{1}$ Karthikeyan Parimelalagapillai* ${ }^{2}$ Sarath Samarage, ${ }^{3}$ Sridharan Sathasivam. ${ }^{1}$ Postgraduate Institute of Medicine, Sri Lanka; ${ }^{2}$ Institute for Health Policy, Sri Lanka; ${ }^{3}$ Ministry of Health, Sri Lanka

10.1136/leader-2021-FMLM.25 